We describe a case of a 3-year-old girl with bilateral pontine and cerebellar infarction due to basilar artery thrombosis, related to a diagnosis of exclusion of severe iron deficiency anaemia who was successfully treated by mechanical thrombectomy.

Case Report Our patient presented with a one-day history of vomiting, and headache, on a background history of a viral prodrome the preceding week, and varicella infection three months earlier. Her haemoglobin was $4.6 \mathrm{~g} / \mathrm{dL}$, with a profoundly microcytic, hypochromic blood film. She received a blood transfusion with clinical improvement. At 36 hours after admission, she became irritable and developed a left divergent squint (NIHSS score 4). Neuroimaging demonstrated acute infarction of the pons, cerebellum and punctate lesions in both occipital lobes. MRA showed complete occlusion of right vertebral artery and basilar artery. Enoxaparin was commenced, but 12 hours later she developed left CNVII palsy and a dense left hemiparesis with hypertension. CT Brain showed worsening ischaemia. Given her ongoing clinical deterioration with major risk of significant morbidity and indeed mortality she underwent mechanical thrombectomy. Successful recannalisation of the basilar artery occurred with a distal left parieto-occipital thrombus remaining. At 9 months follow-up she has a mild left hemiparesis, left CN VII, III and IV palsies and right CNVI palsy, but mobilises independently and is on a normal diet (mRs 2); imaging shows established pontine infarction with gliosis.

Conclusion This case adds to the limited reports of mechanical thrombectomy in children in posterior circulation AIS, as a safe and effective treatment. It also highlights the importance of recognising severe iron-deficiency anaemia as a cause for AIS in children.

\section{GP234 THROMBOLYSIS OF ARTERIAL ISCHAEMIC STROKE IN AN EIGHT MONTH OLD: THE FIRST IRISH CASE}

Noelle Enright*, Laura Melody, Michael Barrett, David Rea, Mary O’Regan. Our Ladies Children's Hospital Crumlin, Dublin, Ireland

\subsection{6/archdischild-2019-epa.293}

Paediatric arterial ischaemic stroke (AIS) is a potentially fatal condition with serious long term neurological sequelae. The rate of paediatric ischaemic stroke is 1.6 - 3.3 per 100,000 and $61 \%$ of these children are left with long term neurological difficulties. Thrombolysis guidelines for children were introduced in May 2017 by the Royal College of Paediatrics and Child Health (RCPCH). However, these guidelines were developed for children from the age of eight and up, and may be used on a case by case basis for children from the age of two to eight years. There is very limited data on the use of thrombolysis in children of any age, particularly in the under two age group. Here we present the first case of AIS thrombolysis in an under two year old in Ireland.

An eight-month old boy presented with a two-and-a-halfhour history of decreased movement of his left side. Importantly, his mother had noted the exact time symptoms commenced. His brain imaging showed a large right sided area of ischaemia in the territory of the middle cerebral artery (MCA), and a narrowed right internal carotid artery (ICA); no haemorrhage was seen. He was brought to intensive care and thrombolysis was commenced with alteplase and heparin. Methylprednisolone was also given as the narrowing of the
ICA was felt to be inflammatory. Other investigations, including echocardiogram, homocysteine level, thrombophilia screen and varicella titres, were all negative.

He has recovered well. He is back on full oral feeds and has learned to walk. He is right hand dominant, with no left sided neglect. He has no speech delay and has been discharged by both physiotherapy and occupational therapy.

Enrolling children in randomised controlled trials to assess AIS treatment and outcomes remains extremely difficult, as evidenced by the Thrombolysis in Pediatric Stroke (TIPS) study, which was closed by the National Institute for Health (NIH) after only thrombolysing one patient despite recruiting 43. The issues centre on delays in presentation (usually outside the thrombolysis window), and in delayed recognition of AIS in emergency departments by clinicians. This case highlights the need for continued research in this area as it demonstrates that successful outcomes are possible after thrombolysis of AIS.

\section{GP235 COL4A1 MUTATION INHERITED FROM MATERNAL MOSAICISM IN AN INFANT PRESENTING WITH MICROCEPHALY, HAEMOLYTIC ANAEMIA AND CATARACTS}

Roisin O'Neill*, Olivia O'Mahony, Niamh McSweeney. Paediatric Neurology Department, Cork University Hospital, Cork, Ireland

\subsection{6/archdischild-2019-epa.294}

Introduction COL4A1-related disorders have overlapping signs and symptoms as a result of fragile blood vessels. The COL4A1 gene located on Chromosome 13 is responsible for making the alpha one subunit of type IV collagen. Type IV collagen molecules form complex protein networks, which are the main component of basement membranes surrounding the body's blood vessels. Clinical presentation and the onset of symptoms are widely varied and largely dependent on the phenotype expressed. A number of phenotypes with overlapping features have been described. These include COL4A1 Brain Small Vessel Disease, AD Familial Porencephaly and HANAC(Hereditary Angiopathy with Nepropathy, Aneurysms and Muscle cramps) Syndrome. COL4A1 disorders are inherited in an autosomal dominant manner and the proportion of cases caused by de novo pathogenic variant is estimated to be $27 \%$.

Case presentation A female infant was born at $36+2$ weeks gestation via Emergency LCSC for IUGR and reduced fetal movements. Apgars were normal. BW was $1.88 \mathrm{~kg}\left(2^{\text {nd }}\right.$ centile $)$ and Birth OFC $29.7 \mathrm{~cm}\left(3^{\text {rd }}\right.$ centile). Initial exam was normal and the infant was admitted to the SCBU for Low Birth weight. She developed haemolytic anaemia week two of life and required three RCC transfusions on DOL 18, 26 and 35. Head growth was found to be static on week four of life and she was also noted to have absent red reflexes bilaterally. Cranial Ultrasound showed a large cystic abnormality in the right cerebral hemisphere. MRI Brain showed cystic encephalomalacia with a large right hemispheric cyst, simplified gyral pattern, significant volume loss and evidence of previous haemorrhage in left cerebral hemisphere. Opthamology confirmed bilateral cataracts. Extensive investigations including metabolic screen, infectious workup, Karyotype and and microarray were all normal. Further genetic testing revealed that the child was heterozygous for novel COL4A1 missence 
variant consistent with a diagnosis of COL4A1-related disorder. Genetic testing of the parents showed that mum had low level mosaicsm, approximately $27 \%$ in leukocyte DNA for the COL4A1 Missense variant.

Conclusion This is the first case of COL4A1 related disorder due to mosacism. Prevalence of COL4A1-related disorder are difficult to establish as fewer than 100 families have been described in the literature. It is likely they are underestimated because of their multisystem and variable phenotype. The significance of the mosaic variant is unknown and mum will be followed up in the adult neurology services to assess for both clinical and radiological features suggestive of subclinical disease.

\section{GP236 FETAL SURGERY FOR SPINA BIFIDA A NEW DEVELOPMENT IN THE IRISH SETTING}

${ }^{1}$ John Joyce*, ${ }^{2}$ Sieglinde Mullers, ${ }^{2}$ Fergal Malone, ${ }^{1}$ Michael Boyle. ${ }^{1}$ Department of Neonatology, Rotunda Hospital, Dublin, Ireland; ${ }^{2}$ Department of Fetal Medicine, Rotunda Hospital, Dublin, Ireland

\subsection{6/archdischild-2019-epa.295}

Introduction Neural tube defects occur in approximately 1/1000 live births in Ireland, with myelomeningocele being the most common form. The current standard management in Ireland involves postnatal closure however access to fetal surgery services is increasing. The Management of Myelomeningocele Study (MOMS) compared prenatal and postnatal closure and showed reduced risk of death, decreased need for ventriculo-peritoneal shunting and improved motor function in the prenatal closure group. We present a case of fetal closure of myelomeningocele referred from our institution.

Clinical Case A 33-year-old female presented to the Rotunda for antenatal care in her second pregnancy following in vitro fertilisation abroad. Her first child was born with myelomeningocele and is a wheelchair user. She had been taking folic acid before and throughout this pregnancy. Antenatal ultrasound revealed a lemon-shaped head with Arnold-Chiari malformation, ventriculomegaly and myelomeningocele at L5/S1. Karyotype and microarray from amniocentesis were normal. She was referred to UCH London for fetal surgery consideration and ultimately underwent hysterotomy and closure of fetal myelomeningocele S1-S3 in Leuven, Belgium at 26 weeks gestation. The procedure involved successful closure of the dura and fascial layers, however, primary closure of the skin was not possible and so a silicone dermal regeneration template was applied to the defect. Improvement in head shape, ventricular size and Chiari malformation were noted on subsequent fetal ultrasound throughout the pregnancy.

The patient presented with abdominal pain at $35+5$ weeks gestation and proceeded to caesarean section. A live born male weighing $3.1 \mathrm{~kg}$ was delivered. He was vigorous with good lower limb movements and anal tone. The silicone graft was in position on the sacrum at delivery and peeled away spontaneously revealing a $2 \mathrm{~cm}$ by $2 \mathrm{~cm}$ patch of healthy granulating skin. There was no defect or leakage of cerebrospinal fluid. A silicone based wound dressing was applied and the infant was admitted to NICU for management. Enteral feeds were quickly established and postnatal cranial ultrasound was normal. He was transferred to Children's University Hospital for ongoing multidisciplinary team input.

Discussion This case represents the second patient to undergo fetal surgery for spina bifida from our institution, in a new partnership with UCH London and Leuven, Belgium. While the surgery is associated with risks of preterm delivery, intraoperative complications and uterine rupture, the potential for significantly better postnatal outcomes make it an important treatment option for some families and should be considered for discussion in suitable cases as they arise.

\section{GP237 THE USE OF MASSAGE THERAPY FOR TREATMENT OF NEONATAL HYPERBILIRUBINEMIA: A SYSTEMATIC REVIEW AND NETWORK META-ANALYSIS}

${ }^{1}$ Mohammed Abdelrahman*, ${ }^{2}$ Nguyen Lam Vuong, ${ }^{3}$ Gehad Tawfik, ${ }^{4}$ Do Phuc Nhu Nguyen, ${ }^{5}$ Le Van Thanh, ${ }^{6}$ Muhammed Elfaituri, ${ }^{7}$ Marwa Mansour, ${ }^{2}$ TB Thoa Le, ${ }^{8}$ Marwa Zaki, ${ }^{9}$ Kenji Hirayama, ${ }^{9}$ Nguyen Tien Huy. ${ }^{1}$ Al-Adan hospital, Kuwait, Kuwait; ${ }^{2}$ University of Medicine and Pharmacy at Ho Chi Minh City, Ho Chi Minh City, Vietnam; ${ }^{3}$ Faculty of Medicine, Ain Shams University, Cairo, Egypt; ${ }^{4}$ Epidemiology Department, The Institute of Public Health Ho Chi Minh City, Ho Chi Minh City, Vietnam; ${ }^{5}$ Faculty of Medicine, University of Medicine and Pharmacy at Ho Chi Minh City, Ho Chi Minh City, Vietnam; ${ }^{6}$ Faculty of Medicine, University of Tripoli, Tripoli, Libya; ${ }^{7}$ Ministry of Health and Population, Cairo, Egypt; ${ }^{8}$ Faculty of Clinical Pharmacy, Fayoum University, Fayoum, Egypt; ${ }^{9}$ Department of Clinical Product Development, Institute of Tropical Medicine (NEKKEN), School of Tropical Medicine and Global Health, Nagasaki University, Nagasaki, Japan

\subsection{6/archdischild-2019-epa.296}

Background Neonatal jaundice is expected to be seen between $(60-80) \%$ of healthy newborns. Massage therapy has been practiced for centuries to gain health benefits in infants. Our aim is to evaluate the effectiveness of various types of infant massage in the reduction of neonatal jaundice and to decrease the need for phototherapy (PT).

Methods An electronic databases search was conducted, with inclusion of studies that investigated newborn babies receiving massage therapy with documented total bilirubin before and after the treatment. Study protocol was registered on PROSPERO, CRD42016049025. Quality assessment was performed using NIH risk of bias tool. Frequentist network meta-analysis (NMA) and meta-analysis (MA) were used to compare all outcomes by each day of follow-up.

Results With 690 studies initially identified, 32 were eligible for qualitative analysis and 29 for quantitative meta-analysis. For neonates requiring PT, our NMA results showed that in 3rd day of life, massage and phototherapy (MP) was significantly the most effective in reduction of serum bilirubin level, while acupressure massage and phototherapy (AMP) was the least compared to by phototherapy only (P), with $\mathrm{MD}, 95 \%$ CI $(-1.16$ [-1.61; -0.72$], 1.28[0.79 ; 1.77]$ respectively). In 14 th day of life, bilirubin level decreased the most in massage with enema and phototherapy (MEP), while MP has no significant difference compared to P. Our MA results in 4th day of life, indicated that MP had a significant decrease in bilirubin level compared with $\mathrm{P}$, with MD 95\%CI (-2.23 [-3.43; 1.03]). In summary comparison with $P, M P$ was the most in decreasing bilirubin level in most of days, with the biggest decrease being in 4th day, while MEP was significantly the most in 14th day. For neonates not requiring PT, our NMA showed a significant decrease in bilirubin with massage and bathing, acupressure massage, and massage 3-4 times/days (M2) groups in 3rd day of life, and with massage 1-2 times/ days (M1) and M2 groups in 4th day. MEP group had a significantly longer duration of PT compared with P, with MD 95\%CI $(29.09$ [9.76; 48.41]). M1 and M2 did not have a significant effect on the requirement for PT. 